\title{
Health-Related Quality of Life in Chinese Patients with Type 2 Diabetes: An Analysis of the Joint Asia Diabetes Evaluation (JADE) Program
}

Andrea OY Luk ${ }^{1,2^{*}}$, Yuying Zhang ${ }^{1}$, Gary TC Ko ${ }^{1,2}$, Nicola Brown ${ }^{1}$, Risa Ozaki ${ }^{2,5}$, Peter CY Tong ${ }^{6}$, Ronald CW Ma ${ }^{3}$, C C Tsang ${ }^{8}$, Y Cheung ${ }^{7}$, Alice PS Kong ${ }^{3}$, C C Chow ${ }^{2}$, Harriet Chung ${ }^{5}$, Maggie Lau ${ }^{8}$, Marina Cheung ${ }^{6}$, Rebecca Wong ${ }^{2}$, Troels Wolthers ${ }^{9}$, Greg Lyubomirsky ${ }^{9}$, Wing-yee So ${ }^{2}$ and Juliana CN Chan ${ }^{1,3,4,5}$, On behalf of the Joint Asia Diabetes Evaluation (JADE) Hong Kong Study Group

${ }^{1}$ Asia Diabetes Foundation, Hong Kong

${ }^{2}$ Department of Medicine and Therapeutics, Prince of Wales Hospital, Shatin, Hong Kong

${ }^{3}$ Department of Medicine and Therapeutics, The Chinese University of Hong Kong, Hong Kong

${ }^{4}$ Hong Kong Institute of Diabetes and Obesity, The Chinese University of Hong Kong, Hong Kong

${ }^{5}$ Yao Chung Kit Diabetes Risk Assessment Centre, Hong Kong

${ }^{6}$ Qualigenics Diabetes Centre, Hong Kong

${ }^{7} \mathrm{Ma}$ On Shan Outpatient Clinic, Hong Kong

${ }^{8}$ Alice Ho Nethersole Hospital, Hong Kong

${ }^{9}$ Merck Sharpe Dohme, USA

\begin{abstract}
Objective: Diabetes is associated with impaired health-related quality of life (HRQLL) which predicts adverse clinical outcome. Our objective was to examine clinical factors associated with HRQoL in a cross-sectional cohort of 14,826 Hong Kong Chinese patients with type 2 diabetes receiving outpatient care.

Methods: Adult patients with type 2 diabetes who underwent comprehensive assessment of risk factors and complications using the web-based Joint Asia Diabetes Evaluation (JADE) Program also completed the EuroQol-5D (EQ-5D) questionnaire and visual analogue scale (VAS) for evaluation of HRQoL. Multiple linear regression analysis was used to identify clinical correlates with EQ-5D index and the association was expressed using $\beta$-coefficient whereby $\beta>0$ indicates positive correlation and $\beta<0$ indicates negative correlation.

Results: More patients reported problems with pain/discomfort $(24.8 \%)$ and anxiety/depression $(20.3 \%)$ than other dimensions of mobility $(7.1 \%)$, self-care $(2.2 \%)$ and usual activities $(4.3 \%)$. Age $(\beta=-0.001)$, female gender $(\beta=-$ $0.049)$, obesity $(\beta=-0.007)$, hypoglycemia at least once monthly $(\beta=-0.04)$, presence of cardiovascular disease $(\beta=-$ $0.034)$, nephropathy $(\beta=-0.014)$ and sensory neuropathy $(\beta=-0.063)$ were independently correlated with lower $E Q-5 D$ index, while hypertension $(\beta=0.017)$ and use of insulin $(\beta=0.017)$ were correlated with higher $E Q-5 D$ index $(p<0.05)$.
\end{abstract}

Conclusion: In Hong Kong Chinese patients with type 2 diabetes, somatic and psychological complaints were common. Apart from demographic characteristics, risk factors, complications and treatment all influenced HRQoL.

Keywords: Health-related quality of life; Type 2 diabetes

\section{Introduction}

The prevalence of diabetes is rising globally and it is estimated that over $60 \%$ of people with diabetes reside in Asia [1]. In a recent population survey conducted in China, up to $11 \%$ of its 1.3 billion population had diabetes and $50 \%$ had pre-diabetes [2]. Relative to the general population, people affected by diabetes consistently reported diminished health-related quality of life (HRQoL) [3-5]. Apart from disabling complications such as blindness, end-stage renal disease (ESRD) and lower extremity amputation, the complexity of treatment regimen, demands for lifestyle adjustment and treatment-related side-effects interfere with daily living and may adversely affect social and psychological functioning. Besides, impaired HRQoL has been demonstrated to predict mortality and cardiovascular events [6,7].

Despite the growing epidemic of diabetes in Asia, there is a relative paucity of data pertaining to HRQoL in these populations. In Chinese patients with type 2 diabetes, low body mass index (BMI), use of insulin, young age and hypoglycemia had been associated with poor HRQoL [8] while physical activity was protective [9]. Of note, despite the importance of HRQoL, both as an encompassing self-perceived measure of health and a possible prognostic predictor, its assessment is frequently overlooked in clinical practice. In this cross-sectional study, we used EuroQol-5D (EQ-5D) to evaluate HRQoL and identify the clinical correlates with EQ-5D in Hong Kong patients with type 2 diabetes receiving outpatient care.

\section{Methods}

\section{Patients}

The Joint Asia Diabetes Evaluation (JADE) Program is a web-based electronic portal developed by the Asia Diabetes Foundation in 2007 with the two-fold objectives to 1) facilitate structured assessment and management of patients with diabetes, and 2) compile disease registry for benchmarking and quality control. The portal contains templates and protocols for periodic comprehensive assessment of risk factors and diabetes complications, risk stratification based on validated risk equations, automated decision support and individualized reports with visual display of 5 -year probabilities of major clinical events to

*Corresponding author: Andrea OY Luk, Asia Diabetes Foundation, Staff Quarter Block B, 4/F, Shatin, New Territories, Hong Kong SAR, Tel: (852)-26376624; Fax (852)-26323108; E-mail: andrealuk@cuhk.edu.hk

Received January 19, 2014; Accepted February 09, 2014; Published February 14,2014

Citation: Luk AOY, Zhang Y, Ko GTC, Brown N, Ozaki R, et al. (2014) HealthRelated Quality of Life in Chinese Patients with Type 2 Diabetes: An Analysis of the Joint Asia Diabetes Evaluation (JADE) Program. J Diabetes Metab 5: 333. doi:10.4172/2155-6156.1000333

Copyright: ( 2014 Luk AOY, et al. This is an open-access article distributed under the terms of the Creative Commons Attribution License, which permits unrestricted use, distribution, and reproduction in any medium, provided the original author and source are credited. 
inform patients and care providers for personalized care [10]. Since its deployment, the JADE program has been used in multiple Asian regions including Hong Kong, China, India, Korea, Philippines, Singapore, Taiwan, Thailand, and Vietnam. In the current analysis, we included all Hong Kong Chinese patients of age 18 years or above with type 2 diabetes who were enrolled into the JADE Program for clinical assessment between 1 July 2007 and 30 June 2012. Referral sources included hospital and community clinics in both private and public settings as well as by self. The use of the JADE portal for research and publication had been approved by the Joint Chinese University of Hong Kong - New Territories East Cluster Clinical Research Ethics Committee. All participants provided informed written consent for the research team to track and analyze their anonymized data.

\section{Assessment}

All patients underwent detailed clinical assessments including documentation of socio-economic status, medical history, family history, lifestyle factors, hypoglycemia and medication use. Significant medical history of cardiovascular and renal disease was cross-checked with either the referring physician or the Hong Kong Hospital Authority Central Computer System which captured admission to public health facilities of residents in Hong Kong. Aside from measurement of blood pressures (BP) and anthropometric parameters, patients were examined for the presence of diabetic retinopathy using retinal photography, peripheral sensory neuropathy using graduated tuning fork and monofilament, and peripheral vascular disease. All assessments were carried out by trained nurses while interpretation of retinal photos was undertaken by diabetologists. Cardiovascular disease was defined as history of coronary heart disease, stroke or peripheral vascular disease, the latter defined as non-traumatic lower extremity amputation and/or ankle:brachial ratio less than 0.9 by Doppler ultrasound scan. Lifestyle factors included current and previous use of tobacco/alcohol, selfmonitoring of blood glucose (SMBG), regular physical activity and adherence to healthy diet within the last 3 months. Hypoglycemia was assessed by asking patients if they have experienced hypoglycemia at least once monthly over the previous three months. Blood and urine samples were collected for plasma glucose, glycated hemoglobin $\left(\mathrm{HbA}_{1 c}\right)$, total cholesterol, low-density lipoprotein (LDL)-cholesterol, high-density lipoprotein (HDL)-cholesterol, triglyceride, renal function test and urine albumin-to-creatinine ratio (ACR), after at least 8 hours of fasting. Hypertension was defined as systolic $\mathrm{BP} \geq 130 \mathrm{mmHg}$, diastolic $\mathrm{BP} \geq 80$ $\mathrm{mmHg}$ or concurrent use of anti-hypertensive drugs. Dyslipidemia was defined as either LDL-cholesterol $\geq 2.6 \mathrm{mmol} / \mathrm{L}$, HDL-cholesterol $<1.0$ $\mathrm{mmol} / \mathrm{L}$, triglyceride $\geq 2.3 \mathrm{mmol} / \mathrm{L}$ or concurrent use of lipid regulating drugs. Obesity was defined by $\mathrm{BMI} \geq 25.0 \mathrm{~kg} / \mathrm{m}^{2}$. Estimated glomerular filtration rate (GFR) as expressed in $\mathrm{ml} / \mathrm{min} / 1.73 \mathrm{~m}^{2}$ was calculated using the abbreviated Modification of Diet in Renal Disease (MDRD) equation recalibrated for Chinese: Estimated GFR $=186 \times[$ SCR $\times 0.011]$ ${ }^{-1.154} \times$ [age $]^{-0.203} \times[0.742$ if female $] \times 1.233$, where SCR was serum creatinine in $\mu \mathrm{mol} / \mathrm{l}$ and 1.233 was the adjusting coefficient for Chinese [11]. Chronic kidney disease (CKD) was defined as estimated GFR (eGFR) $<60 \mathrm{ml} / \mathrm{min} / 1.73 \mathrm{~m}^{2}$ while end-stage renal disease (ESRD) was defined as eGFR $<15 \mathrm{ml} / \mathrm{min} / 1.73 \mathrm{~m}^{2}$ or requirement of renal transplant or dialysis. Microalbuminuria was defined as urine ACR $\geq 2.5-25.0$ $\mathrm{mg} / \mathrm{mmol}$ in men and $\geq 3.5-25$ in women and macroalbuminuria, urine ACR $\geq 25.0 \mathrm{mg} / \mathrm{mmol}$. Nephropathy was defined as either albuminuria or chronic kidney disease.

The EQ-5D is a generic measurement of HRQoL that has been employed in patients with diabetes $[4,12]$. The questionnaire is selfadministered and consists of five dimensions: mobility, self-care, usual activities, pain/discomfort, and, anxiety/depression. Each dimension has three levels: "no problems", "moderate problems", and, "extreme problems". The EQ-5D index was calculated using the United Kingdom (UK) tariff as Hong Kong local tariff is not available [13]. An index of 1 represents full health, 0 represents death, while the lowest possible score of -0.594 indicates health state worse than death. On the visual analogue scale (VAS), patients were asked to rate their health ranging from 0 (worst possible health) to 100 (best possible health). All patients completed the traditional Chinese version of EQ-5D and VAS.

\section{Statistical analysis}

For descriptive analysis, continuous variables were expressed as mean \pm standard deviation (SD) or as median (inter-quartile range), and categorical variables were expressed as percentages. For each EQ5D dimension, the two levels of "moderate problems" and "extreme problems" were combined to form one category: "any problems". The frequency of reporting "any problems" in each dimension was compared across different patient subgroups using Chi-square test. Student t-test or one-way ANOVA was used for the comparison of the EQ-5D index and VAS. Association between EQ-5D utility score and the VAS score was expressed by Spearman correlation coefficient.

We used univariate logistic regression analysis to examine the associations of demographic factors (age, gender, education level), risk factors (duration of diabetes, $\mathrm{HbA}_{1 \mathrm{c}}$, obesity, hypertension, dyslipidaemia), hypoglycemia in previous 3 months, current use of insulin, self-care (SMBG, diet, physical activity, use of tobacco), and presence of complications (nephropathy, retinopathy, sensory neuropathy and cardiovascular-renal disease) with problems in each of the five EQ-5D dimensions. Employment status was not included as employment was strongly correlated with age. Factors nominally significant in the univariate analysis $(p<0.10)$ were selected to enter the multiple logistic regression model to examine for independent associations.

We further examined the association of clinical factors with overall HRQoL as expressed by the EQ-5D index and VAS using multiple linear regression with the backward elimination procedure. The association was expressed using $\beta$-coefficient, a vector which indicates the direction of association such at $\beta>0$ denotes positive correlation between a clinical variable and EQ-5D index / VAS, while $\beta<0$ denotes negative correlation between the clinical variable and EQ-5D index / VAS. Threshold for entry and removal of variables was set at $p<0.05$ and $\mathrm{p}>0.10$, respectively. A 2 -sided $p$-value $<0.05$ was considered significant. Statistical analysis was performed using Statistical Package for Social Science software (version 20, Chicago, Illinois, USA).

\section{Results}

Between 1 July 2007 and 30 June 2012, 15,413 patients with diabetes underwent comprehensive assessment. After excluding patients with type 1 diabetes $(n=347)$ and those of non-Chinese ethnicity $(n=240)$, 14,826 patients were eligible for analysis. The cohort had a mean age 59.2 \pm 11.5 years and mean duration of diabetes of $8.9 \pm 8.0$ years, with up to $41 \%$ in active employment (Table 1 ). Over $80 \%$ had either hypertension or dyslipidemia and over half were obese. The proportion of patients suffering at least one microvascular complication or cardiovascular disease was $53.0 \%$ and $21.6 \%$, respectively. The majority of patients were treated with oral anti-diabetic drugs $(72.2 \%)$ and only $10 \%$ were receiving insulin. Occurrence of hypoglycemic event at least once monthly during the last three months was reported in $8.4 \%$ of subjects. Of the five EQ-5D dimensions, more patients reported problems 
Citation: Luk AOY, Zhang Y, Ko GTC, Brown N, Ozaki R, et al. (2014) Health-Related Quality of Life in Chinese Patients with Type 2 Diabetes: An Analysis of the Joint Asia Diabetes Evaluation (JADE) Program. J Diabetes Metab 5: 333. doi:10.4172/2155-6156.1000333

Page 3 of 7

\begin{tabular}{|c|c|c|c|c|}
\hline & Total & Male & Female & $P$ value \\
\hline Number (\%) & 14826 & $8020(54.1)$ & $6806(45.9)$ & \\
\hline Age (years) & $59.2 \pm 11.5$ & $58.3 \pm 11.3$ & $60.2 \pm 11.6$ & $<0.001$ \\
\hline Employed (\%) & 41.7 & 55.3 & 25.7 & $<0.001$ \\
\hline $\begin{array}{l}\text { Education level (\%) } \\
\text { Illiterate or primary } \\
\text { Middle school } \\
\text { Higher school or above }\end{array}$ & $\begin{array}{l}42.3 \\
39.6 \\
18.1\end{array}$ & $\begin{array}{l}30.6 \\
45.8 \\
23.6\end{array}$ & $\begin{array}{l}56.0 \\
32.4 \\
11.6\end{array}$ & $<0.001$ \\
\hline \multicolumn{5}{|l|}{ Clinical characteristics } \\
\hline Duration of diabetes (years) & $7(2,13)$ & $6(2,12)$ & $8(3,15)$ & $<0.001$ \\
\hline Body mass index $\left(\mathrm{kg} / \mathrm{m}^{2}\right)$ & $25.9 \pm 4.3$ & $26.0 \pm 4.0$ & $25.8 \pm 4.5$ & $<0.001$ \\
\hline Waist circumference $(\mathrm{cm})$ & $89.3 \pm 11.2$ & $91.8 \pm 10.8$ & $86.5 \pm 11.0$ & $<0.001$ \\
\hline Systolic blood pressure $(\mathrm{mmHg})$ & $135 \pm 19$ & $134 \pm 18$ & $136 \pm 20$ & $<0.001$ \\
\hline $\mathrm{HbA}_{1 \mathrm{c}}(\%)$ & $7.5 \pm 1.6$ & $7.5 \pm 1.6$ & $7.5 \pm 1.5$ & 0.464 \\
\hline Fasting plasma glucose (mmol/L) & $7.9 \pm 2.6$ & $7.9 \pm 2.6$ & $7.9 \pm 2.5$ & 0.535 \\
\hline LDL-cholesterol (mmol/L) & $2.6 \pm 0.8$ & $2.6 \pm 0.8$ & $2.7 \pm 0.8$ & $<0.001$ \\
\hline Triglyceride (mmol/L) & $1.3(0.9,1.9)$ & $1.3(0.9,1.9)$ & $1.3(1.0,1.9)$ & 0.005 \\
\hline Estimated GFR (ml/min per $1.73 \mathrm{~m} 2)$ & $104.2 \pm 36.3$ & $103.4 \pm 31.0$ & $105.2 \pm 41.7$ & 0.002 \\
\hline Urine albumin-to-creatinine ratio $(\mathrm{mg} / \mathrm{mmol})$ & $1.6(0.6,7.2)$ & $1.4(0.5,6.9)$ & $1.8(0.7,7.5)$ & $<0.001$ \\
\hline \multicolumn{5}{|l|}{ Co-morbitidies and complications } \\
\hline Hypertension (\%) & 80.8 & 80.5 & 81.2 & 0.330 \\
\hline Dyslipidaemia (\%) & 86.9 & 85.4 & 88.6 & $<0.001$ \\
\hline Obesity (\%) & 54.7 & 56.8 & 52.2 & $<0.001$ \\
\hline $\begin{array}{l}\text { Albuminuria (\%) } \\
\text { Microalbuminuria } \\
\text { Macroalbuminuria }\end{array}$ & $\begin{array}{l}24.4 \\
13.2\end{array}$ & $\begin{array}{l}19.9 \\
13.5\end{array}$ & $\begin{array}{l}29.8 \\
12.9\end{array}$ & $\begin{array}{c}<0.001 \\
0.259\end{array}$ \\
\hline Chronic kidney disease (\%) & 9.3 & 8.7 & 10.0 & 0.006 \\
\hline End-stage renal disease (\%) & 0.7 & 0.7 & 0.7 & 0.796 \\
\hline Retinopathy (\%) & 27.3 & 27.2 & 27.5 & 0.665 \\
\hline Sensory neuropathy (\%) & 4.5 & 4.9 & 4.0 & 0.004 \\
\hline Cardiovascular disease (\%) & 21.6 & 24.0 & 18.8 & $<0.001$ \\
\hline Hypoglycaemia at least once monthly (\%) & 8.4 & 8.0 & 8.9 & 0.047 \\
\hline Malignancy (\%) & 4.5 & 3.9 & 5.2 & $<0.001$ \\
\hline \multicolumn{5}{|l|}{ Lifestyle factors } \\
\hline Current or ex-smoker (\%) & 32.2 & 53.7 & 6.9 & $<0.001$ \\
\hline Adherence to diet (\%) & 52.9 & 50.2 & 56.1 & $<0.001$ \\
\hline Physical exercise at least 3 times per week (\%) & 47.9 & 47.0 & 49.0 & 0.019 \\
\hline Blood glucose monitoring at least once per month (\%) & 62.2 & 61.7 & 62.8 & 0.171 \\
\hline \multicolumn{5}{|l|}{ Treatment } \\
\hline Diet control only (\%) & 17.8 & 19.0 & 16.4 & $<0.001$ \\
\hline Oral hypoglycaemic agent only (\%) & 72.2 & 70.8 & 73.8 & $<0.001$ \\
\hline Insulin (\%) & 10.0 & 10.2 & 9.8 & 0.470 \\
\hline
\end{tabular}

Data are expressed as mean \pm standard deviation, median (inter-quartile range) or percentages as appropriate.

GFR: Glomerular Filtration Rate; HbA1c: Glycated Haemoglobin; LDL: Low-Density Lipoprotein; VAS: Visual Analogue Scale

Table 1: Clinical characteristics of 14,826 Chinese patients with type 2 diabetes.

with pain/discomfort (24.8\%) and depression/anxiety (20.3\%) than problems with mobility, self-care or usual activities. The mean EQ-5D index was $0.897+/-0.173$. The correlation coefficient between EQ-5D index score and VAS was $0.220(\mathrm{p}<0.001)$.

Patients of female gender, older age, unemployed, had long disease duration or obese were more likely to report problems in each of the five EQ-5D dimensions. More patients with microvascular complication or cardiovascular disease reported limitations. Patients who performed frequent SMBG reported more pain/discomfort but no difference on anxiety/depression compared to those who monitored less frequently. Adherence to diet and regular physical activity were both associated with less anxiety/depression (Table 2).
On multiple logistic regression analysis, female gender, sensory neuropathy and cardiovascular disease remained positively correlated with problems in all five dimensions of EQ-5D. Age was correlated with problems in all dimensions except for anxiety/depression which was negatively correlated with age. Obesity was associated with limitation in mobility and pain/discomfort, while $\mathrm{HbA}_{1 \mathrm{c}}$ was associated with anxiety/depression. Occurrence of hypoglycemic events at least once per month was associated with impairment in most domains except self-care (Table 3).

Using multiple linear regression analysis, age, female gender, obesity, occurrence of hypoglycemia at least once monthly, cardiovascular disease, nephropathy and sensory neuropathy were independently correlated with lower EQ-5D index, while hypertension 
Citation: Luk AOY, Zhang Y, Ko GTC, Brown N, Ozaki R, et al. (2014) Health-Related Quality of Life in Chinese Patients with Type 2 Diabetes: An Analysis of the Joint Asia Diabetes Evaluation (JADE) Program. J Diabetes Metab 5: 333. doi:10.4172/2155-6156.1000333

Page 4 of 7

\begin{tabular}{|c|c|c|c|c|c|c|c|}
\hline & \multicolumn{5}{|c|}{ Percentage with problems (\%) } & \multirow[b]{2}{*}{ EQ-5D index } & \multirow[b]{2}{*}{ EQ-5D VAS } \\
\hline & Mobility & Self-care & Usual activities & Pain / discomfort & Anxiety / depression & & \\
\hline Overall with problems & $1049(7.1)$ & $333(2.2)$ & $643(4.3)$ & $3681(24.8)$ & $3012(20.3)$ & & \\
\hline \multicolumn{8}{|l|}{ Gender } \\
\hline Male & 5.4 & 1.8 & 3.3 & 19.4 & 16.0 & $0.920 \pm 0.150$ & $74 \pm 15.1$ \\
\hline Female & $9.0^{\mathrm{a}}$ & $2.7^{\mathrm{a}}$ & $5.6^{\mathrm{a}}$ & $31.2^{\mathrm{a}}$ & $25.4^{\text {a }}$ & $0.870 \pm 0.193$ a & $72.6 \pm 16.2^{\mathrm{a}}$ \\
\hline \multicolumn{8}{|l|}{ Age } \\
\hline $18-40$ years & 2.0 & 0.8 & 2.2 & 16.0 & 16.9 & $0.933 \pm 0.135$ & $74.0 \pm 15.2$ \\
\hline $40-65$ years & 3.9 & 1.2 & 2.6 & 23.6 & 20.9 & $0.905 \pm 0.165$ & $73.3 \pm 15.4$ \\
\hline$>65$ years & $15.4^{\text {a }}$ & $5.0^{\mathrm{a}}$ & $8.8^{\mathrm{a}}$ & $29.0^{\text {a }}$ & $19.5^{b}$ & $0.873 \pm 0.194^{\text {a }}$ & $73.4 \pm 16.2$ \\
\hline \multicolumn{8}{|l|}{ Employment } \\
\hline Employed & 1.8 & 0.3 & 0.9 & 19.5 & 17.3 & $0.928 \pm 0.130$ & $74.3 \pm 14.7$ \\
\hline Unemployed & $10.9^{\text {a }}$ & $3.6^{\mathrm{a}}$ & $6.8^{\mathrm{a}}$ & $28.6^{a}$ & $22.5^{\mathrm{a}}$ & $0.875 \pm 0.195^{a}$ & $72.6 \pm 16.2^{a}$ \\
\hline \multicolumn{8}{|l|}{ Education } \\
\hline$<6$ years & 10.8 & 3.5 & 6.6 & 28.4 & 22.2 & $0.876 \pm 0.195$ & $72.7 \pm 16.6$ \\
\hline $6-11$ years & 4.7 & 1.4 & 2.9 & 23.2 & 19.4 & $0.909 \pm 0.158$ & $73.6 \pm 15.1$ \\
\hline$>11$ years & $3.6^{\mathrm{a}}$ & $1.0^{\mathrm{a}}$ & $2.2^{\mathrm{a}}$ & 20.2 & $17.9^{\mathrm{a}}$ & $0.921 \pm 0.144^{\mathrm{a}}$ & $74.3 \pm 14.1^{\mathrm{a}}$ \\
\hline \multicolumn{8}{|l|}{ Diabetes duration } \\
\hline$<5$ years & 4.6 & 1.4 & 2.8 & 21.2 & 19.7 & $0.910 \pm 0.163$ & $74.0 \pm 15.1$ \\
\hline $5-10$ years & 6.1 & 1.9 & 4.0 & 25.1 & 19.4 & $0.901 \pm 0.170$ & $73.3 \pm 15.6$ \\
\hline$>10$ years & $10.5^{\mathrm{a}}$ & $3.4^{\mathrm{a}}$ & $6.3^{\mathrm{a}}$ & $28.9^{\mathrm{a}}$ & $21.6^{\mathrm{b}}$ & $0.881 \pm 0.186^{a}$ & $72.7 \pm 16.1^{\mathrm{a}}$ \\
\hline \multicolumn{8}{|l|}{$\mathrm{HbA1c}$} \\
\hline$<7.0 \%$ & 7.4 & 2.3 & 4.3 & 24.8 & 20.6 & $0.897 \pm 0.173$ & $74.1 \pm 15.1$ \\
\hline$\geq 7.0 \%$ & 6.6 & 2.2 & 4.4 & 24.8 & 19.9 & $0.898 \pm 0.174$ & $72.8 \pm 15.9^{a}$ \\
\hline \multicolumn{8}{|l|}{ BMI } \\
\hline$<25 \mathrm{~kg} / \mathrm{m} 2$ & 5.9 & 2.3 & 4.0 & 23.3 & 21.0 & $0.900 \pm 0.170$ & $73.7 \pm 15.3$ \\
\hline$\geq 25 \mathrm{~kg} / \mathrm{m} 2$ & $7.7^{\mathrm{a}}$ & 1.9 & 4.3 & $26.0^{\mathrm{a}}$ & $19.7^{\mathrm{b}}$ & $0.898 \pm 0.169$ & $73.1 \pm 15.7^{b}$ \\
\hline \multicolumn{8}{|l|}{ Hypertension } \\
\hline No & 2.3 & 1.1 & 2.0 & 23.6 & 21.4 & $0.902 \pm 0.175$ & $73.4 \pm 15.3$ \\
\hline Yes & $8.2^{\mathrm{a}}$ & $2.5^{a}$ & $4.9^{a}$ & 25.2 & 20.1 & $0.896 \pm 0.173$ & $73.3 \pm 15.7$ \\
\hline \multicolumn{8}{|l|}{ Dyslipidaemia } \\
\hline No & 5.9 & 2.2 & 4.3 & 25.1 & 22.3 & $0.897 \pm 0.167$ & $73.6 \pm 15.3$ \\
\hline Yes & $7.3^{b}$ & 2.3 & 4.3 & 24.8 & $20.0^{\mathrm{b}}$ & $0.897 \pm 0.174$ & $73.3 \pm 15.6$ \\
\hline \multicolumn{8}{|l|}{ Nephropathy } \\
\hline No & 3.9 & 1.3 & 2.5 & 22.8 & 19.5 & $0.909 \pm 0.160$ & $74.1 \pm 15.2$ \\
\hline Yes & $12.0^{\mathrm{a}}$ & $3.7^{\mathrm{a}}$ & $7.2^{\mathrm{a}}$ & $27.9^{\text {a }}$ & $21.5^{\circ}$ & $0.879 \pm 0.190^{a}$ & $72.2 \pm 16.2^{\mathrm{a}}$ \\
\hline \multicolumn{8}{|l|}{ Retinopathy } \\
\hline No & 6.4 & 2.0 & 3.9 & 24.7 & 19.9 & $0.899 \pm 0.172$ & $73.6 \pm 15.4$ \\
\hline Yes & $9.0^{\mathrm{a}}$ & $3.0^{\mathrm{a}}$ & $5.6^{\mathrm{a}}$ & 25.4 & $21.4^{\mathrm{b}}$ & $0.891 \pm 0.176^{c}$ & $72.6 \pm 16.0^{a}$ \\
\hline \multicolumn{8}{|l|}{ Sensory neuropathy } \\
\hline No & 6.3 & 1.9 & 3.9 & 24.3 & 20 & $0.901 \pm 0.168$ & $73.4 \pm 15.5$ \\
\hline Yes & $23.3^{\mathrm{a}}$ & $8.6^{\mathrm{a}}$ & $13.7^{\mathrm{a}}$ & $35.9^{a}$ & $27.2^{\mathrm{a}}$ & $0.815 \pm 0.249^{a}$ & $71.2 \pm 17.4^{a}$ \\
\hline Cardiovascular disea & & & & & & & \\
\hline No & 5.1 & 1.2 & 2.8 & 23.5 & 19.6 & $0.905 \pm 0.162$ & $73.8 \pm 15.5$ \\
\hline Yes & $18.3^{\mathrm{a}}$ & $7.4^{\mathrm{a}}$ & $12.0^{\mathrm{a}}$ & $28.1^{\mathrm{a}}$ & $22.1^{\circ}$ & $0.861 \pm 0.211^{a}$ & $71.9 \pm 16.6^{a}$ \\
\hline 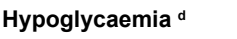 & & & & & & & \\
\hline$<$ once/month & 6.9 & 2.2 & 4.2 & 24.0 & 19.4 & $0.901 \pm 0.171$ & $73.6 \pm 15.5$ \\
\hline$\geq$ once/month & $9.7^{\mathrm{a}}$ & 2.5 & $6.1^{\mathrm{c}}$ & $34.6^{\text {a }}$ & $29.5^{\mathrm{a}}$ & $0.858 \pm 0.197^{\mathrm{a}}$ & $70.8 \pm 16.4^{a}$ \\
\hline Use of insulin & & & & & & & \\
\hline No & 6.9 & 2.2 & 4.3 & 25.2 & 20.2 & $0.897 \pm 0.174$ & $73.7 \pm 15.5$ \\
\hline Yes & 8.2 & 2.4 & 4.4 & $21.8^{\mathrm{c}}$ & 21.7 & $0.902 \pm 0.166$ & $70.3 \pm 16.3^{a}$ \\
\hline Adherence to balance & & & & & & & \\
\hline No & 6.2 & 1.8 & 3.8 & 25.6 & 21.1 & $0.897 \pm 0.172$ & $71.4 \pm 16.1$ \\
\hline Yes & $8.0^{\mathrm{a}}$ & $2.7^{\mathrm{a}}$ & $4.9^{\mathrm{a}}$ & 24.3 & $19.5^{b}$ & $0.897 \pm 0.174$ & $73.5 \pm 15.5^{a}$ \\
\hline Physical exercise ${ }^{e}$ & & & & & & & \\
\hline$<3$ times/ week & 7.9 & 2.8 & 5.4 & 25.2 & 21.0 & $0.892 \pm 0.183$ & $71.9 \pm 15.7$ \\
\hline$\geq 3$ tiems / week & $6.4^{\mathrm{a}}$ & $1.6^{a}$ & $3.3^{a}$ & 24.6 & $19.5^{b}$ & $0.903 \pm 0.163^{a}$ & $74.8 \pm 15.3^{a}$ \\
\hline SMBG ${ }^{e}$ & & & & & & & \\
\hline$<$ once/month & 5.7 & 1.8 & 3.3 & 23.7 & 20.3 & $0.901 \pm 0.171$ & $73.2 \pm 15.8$ \\
\hline$\geq$ once/month & $8.0^{\mathrm{a}}$ & $2.6^{\circ}$ & $5.0^{\mathrm{a}}$ & $25.5^{\mathrm{c}}$ & 20.3 & $0.895 \pm 0.175^{a}$ & $73.5 \pm 15.5$ \\
\hline
\end{tabular}

SMBG: Self Monitoring of Blood Glucose; BMI: Body Mass Index.

${ }^{\mathrm{a}} \mathrm{p}<0.001,{ }^{\mathrm{b}} \mathrm{p}<0.01,{ }^{\mathrm{c}} \mathrm{p}<0.05$, dSelf-reported hypoglycemia within the previous 3 months.

e Adherence to lifestyle factors within the previous 3 months

Table 2:-Percentages of patients with problems in each of the five dimensions of EQ-5D, EQ-5D index and VAS, stratified by clinical characteristics. 
Citation: Luk AOY, Zhang Y, Ko GTC, Brown N, Ozaki R, et al. (2014) Health-Related Quality of Life in Chinese Patients with Type 2 Diabetes: An Analysis of the Joint Asia Diabetes Evaluation (JADE) Program. J Diabetes Metab 5: 333. doi:10.4172/2155-6156.1000333

Page 5 of 7

\begin{tabular}{|c|c|c|c|c|c|}
\hline & \multicolumn{5}{|c|}{ Odd ratio ( $95 \%$ confidence interval) } \\
\hline & Mobility & Self-care & Usual activities & Pain / discomfort & Anxiety / depression \\
\hline Age (10 years) & $1.77(1.65-1.91)^{\mathrm{a}}$ & $1.72(1.52-1.96)^{\mathrm{a}}$ & $1.56(1.43-1.70)^{\mathrm{a}}$ & $1.15(1.10-1.20)^{a}$ & $0.96(0.91-1.00)^{c}$ \\
\hline Female gender & $1.67(1.45-1.93)^{\mathrm{a}}$ & $1.39(1.08-1.77)^{b}$ & $1.65(1.38-1.96)^{a}$ & $1.80(1.65-1.96)^{a}$ & $1.83(1.67-2.00)^{a}$ \\
\hline Disease duration, years & $1.01(1.00-1.02)^{\mathrm{c}}$ & $1.01(0.99-1.02)$ & $1.00(0.99-1.01)$ & $1.01(1.00-1.01)^{\mathrm{c}}$ & $1.00(0.99-1.00)$ \\
\hline Higher education & $0.87(0.68-1.09)$ & $0.76(0.49-1.16)$ & $0.75(0.55-1.01)$ & $0.92(0.81-1.04)$ & $0.92(0.81-1.04)$ \\
\hline Obesity & $1.44(1.25-1.67)^{\mathrm{a}}$ & $0.88(0.69-1.12)$ & - & $1.27(1.16-1.38)^{a}$ & $0.94(0.86-1.03)$ \\
\hline Hypertension & $1.38(1.03-1.84)^{c}$ & $0.85(0.54-1.34)$ & $1.04(0.75-1.43)$ & $0.85(0.76-0.96)^{b}$ & - \\
\hline Dyslipidemia & $0.81(0.65-1.02)$ & - & - & - & $0.81(0.71-0.93)^{b}$ \\
\hline Use insulin & $0.90(0.72-1.14)$ & - & - & - & - \\
\hline $\mathrm{HbA}_{1 \mathrm{c}}$ & - & - & - & - & $1.03(1.00-1.06)^{c}$ \\
\hline Hypoglycemia at least monthly & $1.38(1.10-1.73)^{b}$ & - & $1.31(1.00-1.72)^{\mathrm{c}}$ & $1.70(1.48-1.95)^{a}$ & $1.65(1.43-1.91)^{a}$ \\
\hline Nephropathy & $1.72(1.48-2.01)^{\mathrm{a}}$ & $1.52(1.16-1.99)^{b}$ & $1.73(1.44-2.09)^{a}$ & $1.09(1.00-1.20)$ & $1.02(0.93-1.13)$ \\
\hline Neuropathy & $2.48(1.99-3.09)^{a}$ & $2.16(1.54-3.02)^{a}$ & $2.06(1.59-2.68)^{a}$ & $1.54(1.29-1.83)^{a}$ & $1.50(1.25-1.81)^{\mathrm{a}}$ \\
\hline Diabetic retinopathy & $1.03(0.89-1.20)$ & $1.14(0.89-1.47)$ & $1.04(0.87-1.25)$ & - & $1.07(0.96-1.18)$ \\
\hline Cardiovascular disease & $2.46(2.12-2.85)^{a}$ & $4.08(3.15-5.28)$ & $2.99(2.50-3.58)^{a}$ & $1.14(1.03-1.26)^{b}$ & $1.26(1.13-1.41)^{\mathrm{a}}$ \\
\hline
\end{tabular}

${ }^{\mathrm{a}} \mathrm{p}<0.001,{ }^{\mathrm{b}} \mathrm{p}<0.01,{ }^{\mathrm{c}} \mathrm{p}<0.05$

"-" Not being entered into multivariate regression model (univarate regression $\mathrm{P}>0.1$ )

Table 3: Factors associated with problems in each EQ5D dimension using multivariate logistic regression.

\begin{tabular}{|c|c|c|c|c|c|c|}
\hline & \multicolumn{3}{|c|}{$E Q-5 D$ index } & \multicolumn{3}{|c|}{ VAS } \\
\hline & Coefficients $\beta$ & Standard Error & P-value & Coefficients $\beta$ & Standard Error & P-value \\
\hline Intercept & 0.985 & 0.01 & $<0.001$ & 76.2 & 1.1 & $<0.001$ \\
\hline Age & -0.001 & 0.000 & $<0.001$ & 0.033 & 0.014 & 0.018 \\
\hline Female gender & -0.049 & 0.003 & $<0.001$ & -1.579 & 0.288 & $<0.001$ \\
\hline Disease duration, years & 0.000 & 0.000 & 0.074 & - & - & - \\
\hline Higher education & 0.007 & 0.004 & 0.087 & 0.816 & 0.388 & 0.035 \\
\hline Obesity & -0.007 & 0.003 & 0.023 & -0.534 & 0.293 & 0.068 \\
\hline Hypertension & 0.017 & 0.004 & $<0.001$ & 0.721 & 0.397 & 0.069 \\
\hline Dyslipidemia & 0.008 & 0.005 & 0.072 & - & - & - \\
\hline Use insulin & 0.017 & 0.005 & 0.001 & -2.390 & 0.486 & $<0.001$ \\
\hline $\mathrm{HbA1c}$ & - & - & & -0.396 & 0.094 & $<0.001$ \\
\hline Hypoglycemia $\geq$ monthly & -0.04 & 0.005 & $<0.001$ & -2.585 & 0.507 & $<0.001$ \\
\hline Nephropathy & -0.014 & 0.003 & $<0.001$ & -1.105 & 0.309 & $<0.001$ \\
\hline Neuropathy & -0.063 & 0.007 & $<0.001$ & -1.547 & 0.650 & 0.017 \\
\hline Cardiovascular disease & -0.034 & 0.004 & $<0.001$ & -1.880 & 0.364 & $<0.001$ \\
\hline
\end{tabular}

Backward selection was used.

"- "Not being entered into multivariate regression model (univarate regression $\mathrm{P}>0.1$ )

Table 4: Factors associated with EQ-5D index using the UK tariff of the EQ-5D index and visual analogue scale (VAS) among Hong Kong Chinese patients with type 2 diabetes.

and use of insulin were correlated with higher EQ-5D index. Higher education was marginally associated with higher EQ-5D index but did not reach statistical significance. Disease duration, $\mathrm{HbA}_{1 \mathrm{c}}$ and diabetic retinopathy were not associated with EQ-5D index in this model. Using VAS as the dependent variable, we observed that older age and higher education were both correlated with higher VAS, while use of insulin and high $\mathrm{HbA}_{1 \mathrm{c}}$ with lower VAS (Table 4).

\section{Discussion}

To our knowledge, this is the largest cross-sectional analysis of HRQoL in Chinese type 2 diabetic patients examining over 14,000 subjects recruited from different clinic settings. Over $20 \%$ of patients reported pain/discomfort and anxiety/depression while mobility, self-care and usual activities were less affected. Apart from old age and female gender, obesity, frequent hypoglycemia and diabetic complications were independently associated with decrement in EQ-5D index while hypertension and use of insulin were associated with better HRQoL.

\section{Diabetes complications and HRQoL}

In this cohort consisting of ambulatory patients, the mean EQ$5 \mathrm{D}$ index was $0.897 \pm 0.173$, which was comparable to a report from Singapore [14]. Most reports from Caucasian diabetic population had lower indices $[15,16]$, probably due to different study designs, settings and patient characteristics. In agreement with most qualitative research, increased age and female gender had worse HRQoL which might reflect genetic, developmental, biological, socio-economical and cultural differences associated with age and gender [15].

Overall, our results accorded with a smaller survey of 206 Chinese type 2 diabetic patients in which complications and hospitalizations for hypoglycemia predicted poor diabetes-specific HRQoL [8]. In our analysis, $50 \%$ of patients had microvascular disease, $20 \%$ had cardiovascular disease, and $10 \%$ had chronic kidney disease, all of whom had worse HRQoL than those without complications. Using the same 
instrument, patients with micro- or macrovascular complications in the United Kingdom Prospective Diabetes Study also had lower EQ-5D indices than those without complications [17]. Of note, nephropathy, neuropathy and cardiovascular disease were independently associated with low EQ-5D index while retinopathy was not. We speculate that as the majority of our patients had non-proliferative retinopathy (data not shown), the lack of visual symptoms with mild eye complication may account for the absence of correlation.

We are not able to fully explain the positive correlation of hypertension with high EQ-5D index. We postulate that excessive lowering of BP might cause adverse symptoms such as dizziness and weakness which may impair HRQoL.

\section{Obesity and HRQoL}

Using Asian definitions of central and general obesity, over 50\% of our patients were obese which was associated with lower EQ-5D index. Obesity, with or without diabetes, is associated with multiple morbidities including all bodily systems and is a recognized factor for poor quality of life [18]. In a large population survey of adults in the United States, overweight subjects reported reduced self-perceived health [18]. In the LookAHEAD trial, subjects who had higher BMI with the lowest exercise capacities scored most unfavorably on physical component on Short Form-36 while those with high BMI but high exercise capacities reported less impairment [19]. These findings suggested that the negative impact of overweight or obesity on HRQoL might be offset by regular physical exercise and improvement in exercise tolerance. In our analysis, although patients who exercised frequently had higher EQ-5D index, frequent exercise was not correlated with the index when adjusted for other clinical factors while the association of obesity with HRQoL remained.

\section{Glycemic control and HRQoL}

Contrary to BMI, $\mathrm{HbA}_{1 \mathrm{c}}$ was not associated with EQ-5D index although it was correlated with VAS. Indeed, the relationship of glycemic status with HRQoL have been inconsistent in most surveys $[8,14,20]$. The EQ-5D index is a composite of pre-defined domains pertaining to specific physical and psychological functioning, and thus may be less sensitive to other aspects of QoL such as psychosocial wellbeing, social support and sense of empowerment which might affect glycemic control. On the other hand, VAS is less restrictive and perhaps better at reflecting overall health status from patients' perspectives. Of note, a previous study conducted in Hong Kong also failed to show an association between $\mathrm{HbA}_{1 \mathrm{c}}$ and HRQoL despite using a diabetesspecific instrument [8].

Strategies to manage diabetes including SMBG and insulin therapy may cause discomfort and consistent with other studies, we found that 1 in 4 patients reported pain/discomfort. Insulin use was associated with higher EQ-5D index but lower VAS, while frequent SMBG with finger pricking was not associated with either on multivariate regression. Given the inter-correlations between $\mathrm{HbA} \mathrm{Ac}_{1 \mathrm{c}} \mathrm{SMBG}$ and insulin use, it is plausible that some of the negative effects associated with physical discomfort due to insulin injection and SMBG, might be countered by increased energy level associated with improved glycemic control. In a large longitudinal study evaluating the effect of diabetes education, participation in education program resulted in increased uptake of insulin use and self-care activities, increased rates of reaching metabolic goals, and importantly, lower rates of vascular complications and possibly improvement in work productivity [21]. In this way, pain / discomfort aside, improvement in glycemic control and reduced risks of diabetes complications following insulin use may in part explain the positive association of insulin therapy and EQ-5D index.

\section{Hypoglycemia and HRQoL}

After age, female gender and complications, frequent hypoglycemia had the largest effect size on multiple dimensions of EQ-5D, notably pain/discomfort and anxiety/depression. In a separate cross-sectional study conducted in the Asia-Pacific, hypoglycemic symptoms were correlated with anxiety/depression, pain/discomfort and problems with usual activities [22]. Previously considered to be exclusive to insulin users, hypoglycemia is common even among non-insulin treated patients with $8.4 \%$ of the present cohort reporting hypoglycemia more than once per month over the previous three months. In addition to the immediate negative experience, patients might develop fear and anxiety over recurrent events. Our findings emphasized the importance of individualizing treatment goal and personalizing care in order to avoid hypoglycemia [23]

\section{Anxiety and depression}

In this survey, up to 1 in 5 patients had anxiety/depression. In a subset of patients from this cohort, using the Patient Health Questionnaire (PHQ9), a validated tool for depression screening [24], we have also reported similar proportions of patients with negative emotions. The bidirectional associations between diabetes and depression is being increasingly recognized [25,26] although the underlying nature requires elucidation. While depression might lead to lethargy, poor self-care and suboptimal risk factor control, treatment demands and psychological burden on future risk of serious complications might also cause negative emotions. In the multinational Diabetes Attitudes, Wishes, and Needs (DAWN) study [27], over $20 \%$ of diabetic patients had psychological distress or depression. In agreement with other studies $[15,28]$, we found that while increased age was correlated with problems in domains pertaining to physical function, young age was correlated with anxiety/depression. Herein, young adults with chronic diseases often had lower socioeconomic status, poorer social support and social stigmata. Besides, these subjects might be less emotionally prepared to cope with a lifelong illness than elderly, who were more ready to accept their conditions and maintain better patient-carer relationship [29].

\section{Limitations}

Our study had a number of limitations. First, the cross-sectional nature of the present study precluded examination of causality. Second, the EQ-5D was not diabetes-specific but a generic instrument for measuring HRQoL. As such, it might not have sufficiently measured the multiple physical, psychological and behavioral problems associated this complex condition, such as stress related to self-management, polypharmacy and restriction on lifestyle. The emphases of EQ-5D on mobility, self-care and usual activities, which are more relevant to subjects with physical disabilities, might overestimate HRQoL in this outpatient population with relatively young age. Third, since the Hong Kong EQ-5D tariff is not available, we used the UK tariff which was chosen for two reasons. One, both areas had a predominantly public-funded health care system and two, Hong Kong which has been governed by UK for over a century is a metropolitan city with a relatively westernized culture.

\section{Conclusions}

In this large Chinese cohort with type 2 diabetes, $20 \%$ of patients had pain/discomfort and anxiety/depression. Age, female gender, 
Citation: Luk AOY, Zhang Y, Ko GTC, Brown N, Ozaki R, et al. (2014) Health-Related Quality of Life in Chinese Patients with Type 2 Diabetes: An Analysis of the Joint Asia Diabetes Evaluation (JADE) Program. J Diabetes Metab 5: 333. doi:10.4172/2155-6156.1000333

obesity, complications and hypoglycemia were associated with impaired HRQoL. While old patients more frequently reported physical discomfort, anxiety/depression was associated with younger age. These complex associations pertaining to demographics, risk factor control and treatment called for systematic evaluation of HRQoL to identify special needs in different subgroups for personalized, pharmacological and non-pharmacological interventions.

\section{Acknowledgement}

We would like to thank all nursing and clerical staff at the Yao Chung Kit Diabetes Assessment Centre and Diabetes and Endocrine Centre of the Prince of Wales Hospital for their involvement in patient enrolment and care.

\section{References}

1. International Diabetes Federation (2013) IDF Diabetes Atlas (6thedn) Brussels Belgium.

2. Xu Y, Wang L, He J, Bi Y, Li M, et al. (2013) Prevalence and control of diabetes in Chinese adults. JAMA 310: 948-959.

3. Schunk M, Reitmeir P, Schipf S, Völzke H, Meisinger C, et al. (2012) Healthrelated quality of life in subjects with and without Type 2 diabetes: pooled analysis of five population-based surveys in Germany. Diabet Med 29: 646-653.

4. Grandy S, Fox KM (2008) EQ-5D visual analog scale and utility index values in individuals with diabetes and at risk for diabetes: Findings from the Study to Help Improve Early evaluation and management of risk factors Leading to Diabetes (SHIELD). Health Qual Life Outcomes 6: 18.

5. Sundaram M, Kavookjian J, Patrick JH, Miller LA, Madhavan SS, et al. (2007) Quality of life, health status and clinical outcomes in Type 2 diabetes patients. Qual Life Res 16: 165-177.

6. Kleefstra N, Landman GW, Houweling ST, Ubink-Veltmaat LJ, Logtenberg SJ, et al. (2008) Prediction of mortality in type 2 diabetes from health-related quality of life (ZODIAC-4). Diabetes Care 31: 932-933.

7. Clarke PM, Hayes AJ, Glasziou PG, Scott R, Simes J, et al. (2009) Using the EQ-5D index score as a predictor of outcomes in patients with type 2 diabetes. Med Care 47: 61-68.

8. Shiu AT, Thompson DR, Wong RY (2008) Quality of life and its predictors among Hong Kong Chinese patients with diabetes. J Clin Nurs 17: 125-132.

9. Cong JY, Zhao Y, Xu QY, Zhong CD, Xing QL (2012) Health-related quality of life among Tianjin Chinese patients with type 2 diabetes: a cross-sectional survey. Nurs Health Sci 14: 528-534.

10. Ko GT, So WY, Tong PC, Le Coguiec F, Kerr D, et al. (2010) From design to implementation--the Joint Asia Diabetes Evaluation (JADE) program: a descriptive report of an electronic web-based diabetes management program. BMC Med Inform Decis Mak 10: 26.

11. Ma YC, Zuo L, Chen JH, Luo Q, Yu XQ, et al. (2006) Modified glomerula filtration rate estimating equation for Chinese patients with chronic kidney disease. J Am Soc Nephrol 17: 2937-2944

12. Glasziou P, Alexander J, Beller E, Clarke P; ADVANCE Collaborative Group (2007) Which health-related quality of life score? A comparison of alternative utility measures in patients with Type 2 diabetes in the ADVANCE trial. Health Qual Life Outcomes 5: 21.

13. Dolan P (1997) Modeling valuations for EuroQol health states. Med Care 35 1095-1108

14. Shim YT, Lee J, Toh MP, Tang WE, Ko Y (2012) Health-related quality of life and glycaemic control in patients with Type 2 diabetes mellitus in Singapore. Diabet Med 29: e241-248.

15. Redekop WK, Koopmanschap MA, Stolk RP, Rutten GE, Wolffenbuttel BH, et al. (2002) Health-related quality of life and treatment satisfaction in Dutch patients with type 2 diabetes. Diabetes Care 25: 458-463.

16. Solli O, Stavem K, Kristiansen IS (2010) Health-related quality of life in diabetes: The associations of complications with EQ-5D scores. Health Qual Life Outcomes 8: 18

17. (1999) Quality of life in type 2 diabetic patients is affected by complications but not by intensive policies to improve blood glucose or blood pressure control (UKPDS 37). U.K. Prospective Diabetes Study Group. Diabetes Care 22: 11251136.

18. He XZ, Baker DW (2004) Body mass index, physical activity, and the risk of decline in overall health and physical functioning in late middle age. Am J Public Health 94: 1567-1573.

19. Rejeski WJ, Lang W, Neiberg RH, Van Dorsten B, Foster GD, et al. (2006) Correlates of health-related quality of life in overweight and obese adults with type 2 diabetes. Obesity (Silver Spring) 14: 870-883

20. Goddijn PP, Bilo HJ, Feskens EJ, Groeniert KH, van der Zee KI, et al. (1999) Longitudinal study on glycaemic control and quality of life in patients with Type 2 diabetes mellitus referred for intensified control. Diabet Med 16: 23-30.

21. Gagliardino JJ, Aschner P, Baik SH, Chan J, Chantelot JM, et al (2010) Patients' education, and its impact on care outcomes, resource consumption and working conditions: data from the International Diabetes Management Practices Study (IDMPS). Diabetes Metab 38: 128-134.

22. Sheu WH, Ji LN, Nitiyanant W, Baik SH, Yin D, et al. (2012) Hypoglycemia is associated with increased worry and lower quality of life among patients with type 2 diabetes treated with oral antihyperglycemic agents in the Asia-Pacific region. Diabetes Res Clin Pract 96: 141-148.

23. Raz I, Riddle MC, Rosenstock J, Buse JB, Inzucchi SE, et al. (2013) Personalized management of hyperglycemia in type 2 diabetes: reflections from a Diabetes Care Editors' Expert Forum. Diabetes Care 36: 1779-1788.

24. Zhang Y, Ting R, Lam M, Lam J, Nan H, et al. (2013) Measuring depressive symptoms using the Patient Health Questionnaire-9 in Hong Kong Chinese subjects with type 2 diabetes. J Affect Disord 151: 660-666.

25. Goldney RD, Phillips PJ, Fisher LJ, Wilson DH (2004) Diabetes, depression and quality of life: a population study. Diabetes Care 27: 1066-1070.

26. Egede LE, Ellis C (2010) Diabetes and depression: global perspectives Diabetes Res Clin Pract 87: 302-312.

27. Snoek FJ, Kersch NYA, Eldrup E, Harman-Boehm I, Hermanns N, et al. (2011) Monitoring of Individual Needs in Diabetes (MIND): Baseline data from the cross-national Diabetes Attitudes, Wishes, and Needs (DAWN) MIND Study. Diabetes Care 34: 601-603.

28. Sherbourne CD, Meredith LS, Rogers W, Ware JE Jr (1992) Social support and stressful life events: age differences in their effects on health-related quality of life among the chronically ill. Qual Life Res 1: 235-246.

29. Maddigan SL, Majumdar SR, Johnson JA (2005) Understanding the complex associations between patient-provider relationships, self-care behaviours, and health-related quality of life in type 2 diabetes: a structural equation modeling approach Qual Life Res 14: 1489-1500. 